\title{
Design of the MiniCLEAN dark matter search veto detector subsystem
}

\author{
Robert Abruzzio $^{a}$, Benjamin Buck ${ }^{a *}$, Stephen Jaditz ${ }^{a}$, James Kelsey ${ }^{a}$, \\ Jocelyn Monroe $^{b}$, Kimberly Palladino ${ }^{c}$ \\ ${ }^{a}$ Department of Physics, Massachusetts Institute of Technology, \\ Cambridge, MA, USA \\ E-mail: bbuckemit.edu \\ ${ }^{b}$ Department of Physics, Royal Holloway University of London, \\ Egham, Surrey, $U K$ \\ ${ }^{c} S N O L A B$, \\ Lively, Ontario, $C A$
}

\begin{abstract}
This paper describes the design of the active muon veto subsystem for the MiniCLEAN dark matter direct detection experiment at SNOLAB in Sudbury, Ontario, Canada. The water-filled veto is instrumented with 48 PMTs which are read out by front end electronics to time multiplex 48 photomultiplier channels into 6 digitizer channels and provide an instantaneous hit sum across the subsystem (N-Hit) for the veto trigger. We describe the primary system components: the PMTs, the support structure, the front-end electronics, and the data acquisition system.
\end{abstract}

KEYWORDS: Dark matter detectors, Photon detectors for UV, visible and IR photons (vacuum), Front-end electronics for detector readout.

${ }^{*}$ Corresponding author. 


\section{Contents}

1. Introduction 四

2. Mechanical design of the MiniCLEAN muon veto subsystem 2

2.1 PMTs, connectors, cables

2.2 Mechanical hardware 5

3. Electronics design of the MiniCLEAN veto subsystem

3.1 Amplifier discriminator board 6

3.2 Delay lines

3.3 Summer board 8

3.4 Housing and assembly 8

母. Summary 9

\section{Introduction}

The MiniCLEAN experiment is searching for Weakly Interacting Massive Particle (WIMP) dark matter using a liquid argon target. The MiniCLEAN detector physics goals and design are described in [1]. The detector is located 6800 feet underground in the Cube Hall at SNOLAB in Sudbury, Ontario, Canada. The detector consists of a spherical stainless steel inner vessel containing liquid argon or neon surrounded by 92 cryogenic photomultiplier tubes (PMTs) inside an outer vacuum vessel. The outer vessel is located within a water tank, shown in Figure [1, which provides additional background shielding as well as a veto function for external particles incident on the argon detector volume.

The veto water shields the liquid argon target from low energy neutrons and gamma rays produced by radioactivity in the surrounding cavern rock and tags cosmic muons which penetrate the rock overburden to the detector depth. These muons can create high energy neutrons, which may mimic the WIMP signal. From a simulation of the cosmogenic muon flux, using the Sudbury Neutrino Observatory (SNO) measurement of $3.31 \pm 0.01$ (stat.) \pm 0.09 (sys.) $\times 10^{-10} \mu / \mathrm{cm}^{2} / \mathrm{s}$ [2], and the energy and angular distribution parameterization from [3], the expected total muon flux through the MiniCLEAN veto tank water is 9.8 muons/day. 48 PMTs are used to detect Cherenkov light produced by muons transiting the water, and trigger a veto signal. The veto was designed to tag $99.9 \%$ of cosmic muons, and GEANT4 [四] simulations were done to determine the number and placement of PMTs inside the water volume.

The MiniCLEAN Muon Veto Subsystem consists of:

- 48 PMTs mounted on 12 strings of 4 PMTs around the inner diameter of the MiniCLEAN water shield tank; 
- high voltage power supply for the PMTs; and,

- veto PMT signal electronics, which include custom amplifier discriminator boards, custom summer boards, and one CAEN V1720 digitizer board for data acquisition.

The custom electronics bias the PMTs, time multiplex 8 PMT channels into a single digitizer channel, and generate a signal proportional to the number of PMTs above threshold at any moment which is used as the veto trigger to the DAQ. An overview of the system is shown in Figure 5.

This paper describes the mechanical and electrical design of the veto subsystem. The mechanical system is described in Section 2, and the electronics are described in Section 3 .

\section{Mechanical design of the MiniCLEAN muon veto subsystem}

The water tank for the MiniCLEAN veto is a bottomless silo, with radius $2.8 \mathrm{~m}$ and height $7.9 \mathrm{~m}$. These dimensions provide $1.5 \mathrm{~m}$ or greater water thickness between the cavern air and outer vessel. The veto interior surface is covered by a highly-reflective waterproof liner, with four holes in the bottom through which the supports for the main detector pass into the cavern floor. Four 8-inch Hamamatsu R1408 PMTs from SNO are attached to each of 12 equally spaced poles which hang in the water along the inside wall of the tank. GEANT4 [- 4 ] simulations of the veto performance were used to optimize the mechanical design. All PMTs point inward, normal to the tank wall. The heights of the PMTs are the midpoints of four equal lengths extending along the entire height of the tank. The veto configuration is shown in Figure [1.

The simulation of this geometry and the Hamamatsu R1408 PMT response (described in Section 2.1 results in an average of approximately 25 photoelectrons (p.e.) detected per PMT per muon in the water. The average time spread between first and last detected p.e. of the signal on a given PMT is $\sim 15 \mathrm{~ns}$. The electronics described in Section 3.1 are designed for a 0.25 p.e. threshold $(2.5 \mathrm{mV})$ per PMT; requiring a coincidence of at least 2 PMTs above this threshold (N-Hit $>2)$ results in an efficiency of 0.999 giving 3 un-vetoed muons per year. For comparison, the simulated efficiency drops to 0.99 if the N-Hit threshold is 1 p.e. and the veto trigger requirement is $\mathrm{N}$-Hit $>8$. The un-vetoed muons traverse less than $5 \mathrm{~cm}$ of water, clipping the corners of the veto tank volume. From a simulation of 75 years of cosmogenic muon backgrounds, the estimated number of cosmogenic background induced scatters in the liquid argon target with $20-100 \mathrm{keVee}$ is $<0.1$ year.

\subsection{PMTs, connectors, cables}

66 Hamamatsu R1408 PMTs were made available from SNO for use in the MiniCLEAN veto. The gain and dark rates of these PMTs were measured as a function of bias voltage in a PMT test stand at Los Alamos National Laboratory. We used a CAEN V1720 digitizer with the MiniCLEAN DAQ software, DCDAQ, for data acquisition [5]. PMTs were conditioned at $2100 \mathrm{~V}$ for 12 hours. Next, a single photoelectron gain measurement as a function of bias voltage was made using a blue LED flashed at low intensity, such that PMT occupancy was several percent. Final gain and dark rate measurements were made in a similar test stand at MIT, and 48 PMTs plus 5 spares were selected to use in the veto. The selection criterion was to chosen to minimize dark rate. We required the rate of pulses with $>3 \mathrm{mV}$ (approximately 0.3 p.e.) to be less than $3000 \mathrm{~Hz}$ in the absence of a light source. Operating voltages were chosen to gain match the PMTs for an anode gain of $1 \times 10^{7}$; the 


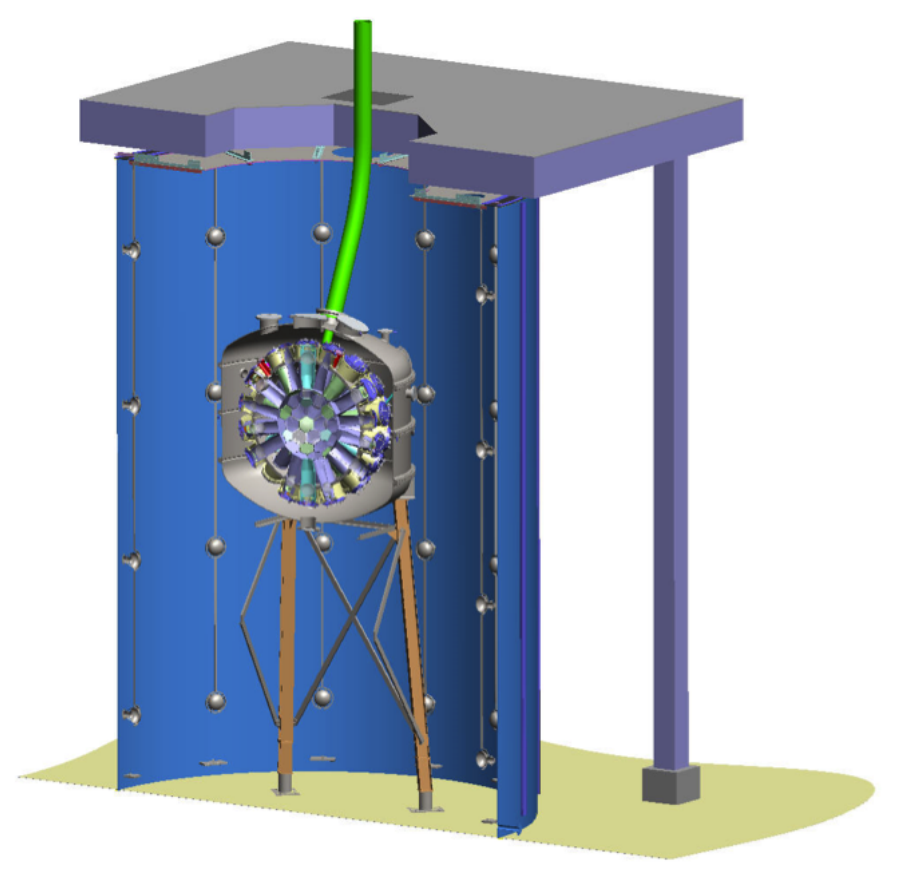

Figure 1. Model of MiniCLEAN veto showing the shield tank, inner liquid argon detector, and veto PMTs.

range of operating voltages is $1800-2300 \mathrm{~V}$. A typical single p.e. pulse charge is $\sim 1 \mathrm{pC}$, with pulse amplitude of $\sim 10 \mathrm{mV}$ and pulse full width of $\sim 10 \mathrm{~ns}$. These PMTs were also qualified in tests done many years ago for the Sudbury Neutrino Observatory (SNO), and the optimum operating voltage was found to be consistent with these earlier measurements. An example PMT single p.e. charge spectrum at its operating voltage is shown in Figure 2 .

The SNO PMT bases are fitted with custom, waterproof TNC-type jack connectors which mate 75 Ohm RG59 cable to the PMTs. These custom connectors have been built by the MIT machine shop from the SNO-TRIUMF design. An assembly drawing of the connector is shown in Figure 3 along with a picture of one of those built at MIT. The MIT-built SNO connectors were prototyped, tested at voltage in air, and tested at voltage in water before the full connector complement was machined. After cable assembly, all of the machined connectors were tested in a test stand at RHUL in water at voltage with PMTs attached. All of the PMTs were tested in water at voltage with the MIT-built connector sample. Several failures of cable connectors were observed in dry tests. These experiences were used to modify the cable assembly procedure after which no cable connectors or PMTs were found to fail these wet tests.

The connection to the PMTs uses a custom cable, Belden part number YR29304 Rev 2. The cable is a RG-59 coaxial cable and is the same cable used by SuperK and SNO. The cable is flooded, has a waterproof jacket and is rated to $2.3 \mathrm{kV}$.

The SNO PMTs come with waterproof base enclosures, consisting of a plastic cylinder filled with gel. All of the PMTs were immersed in water and operated at voltage for approximately 4 hours in a test stand at MIT to look for base waterproofing or connector failures. However, this test did not simulate the water pressure at the bottom of the veto tank. To address this question, 


\section{PAYG at $1882 \mathrm{~V}$}

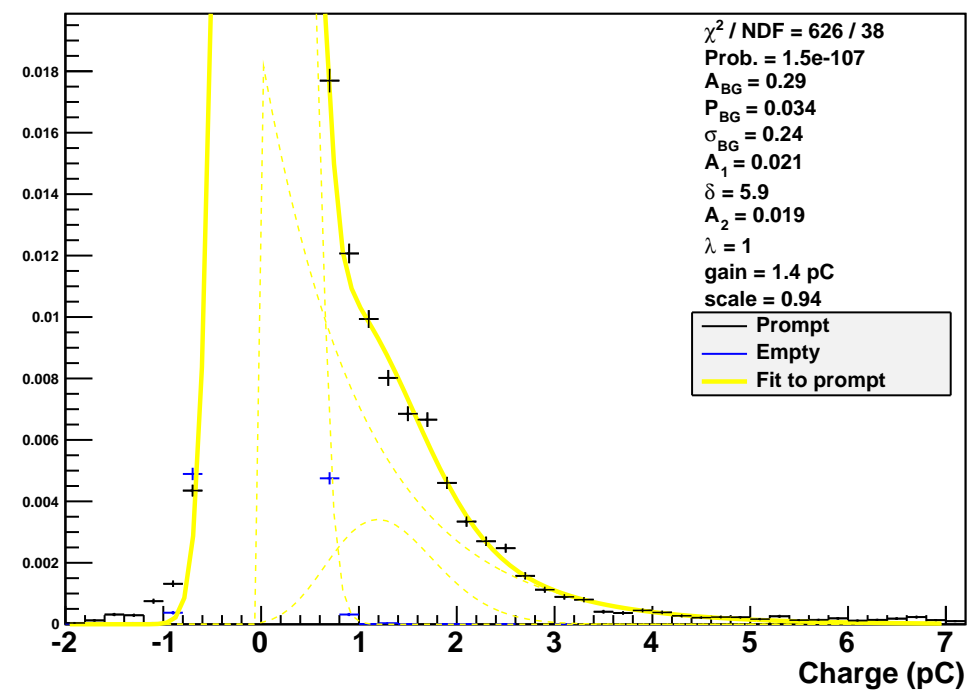

Figure 2. Single photoelectron charge spectrum for PMT ID PAYG at operating voltage. The data with a pulsed LED are shown in black, with statistical errors, noise data are shown in blue, and the fit model is shown in the yellow line.

a pressure vessel was assembled at MIT Bates Research and Engineering Center to test the PMT bulbs, base enclosures, connectors, and cables for waterproofness and structural integrity at pressure equivalent to that at the bottom of the veto. Testing all of the PMTs at atmospheric pressure was successful and a random subset of $10 \%$ of the PMTs tested at 4 atm for several weeks (a factor of 2 above the maximum pressure in the MiniCLEAN veto tank) found no failures. Therefore we concluded that further encapsulation of the SNO waterproof base enclosure was not necessary.
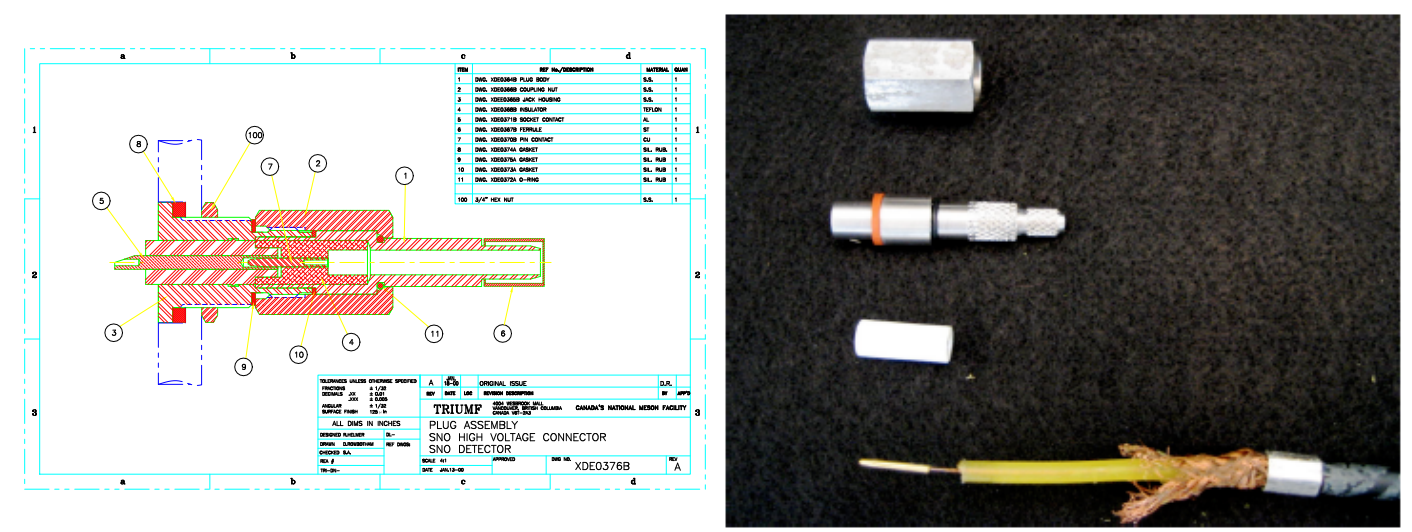

Figure 3. Left: TRIUMF connector assembly drawing. Items 1,2,4,6,7,10, and 11 were built or purchased to mate RG59 cable to the existing R1408 PMT jacks. Right: As-built TRIUMF connector for mating cable to the R1408 jack. From top to bottom is shown the coupling nut, the plug body into which the cable is inserted, the teflon insulator which is also inserted into the plug body, and the pin with a stripped cable. 


\subsection{Mechanical hardware}

The PMT support structures attach the PMTs to poles or "strings" which attach to the veto tank lid. These have been built at MIT Bates Research and Engineering Center. The 316L stainless steel mount and string are shown in Figure $\emptyset$. The Buna-N rubber pads contacting the base enclosure and bulb accommodate the variations in dimensions across the set of PMTs. The base enclosures' and bulbs' diameters were measured to vary between 3.00 and 3.34 inches and between 7.91 and 8.02 inches. The rubber mounting pads were all fit by hand to each PMT at Bates before shipment to SNOLAB and tested with a load of twice the buoyant force (maximum $8 \mathrm{lbs} / \mathrm{PMT}$ ) to try to dislodge the PMT from the mount. This procedure will be repeated during assembly to verify the mechanical stability of each veto PMT in its mount. These tests were done with both wet and dry rubber pads. A complete assembly test of one string, consisting of four PMTs and associated hardware, dry, was done Bates (shown in Figure 4 , right).
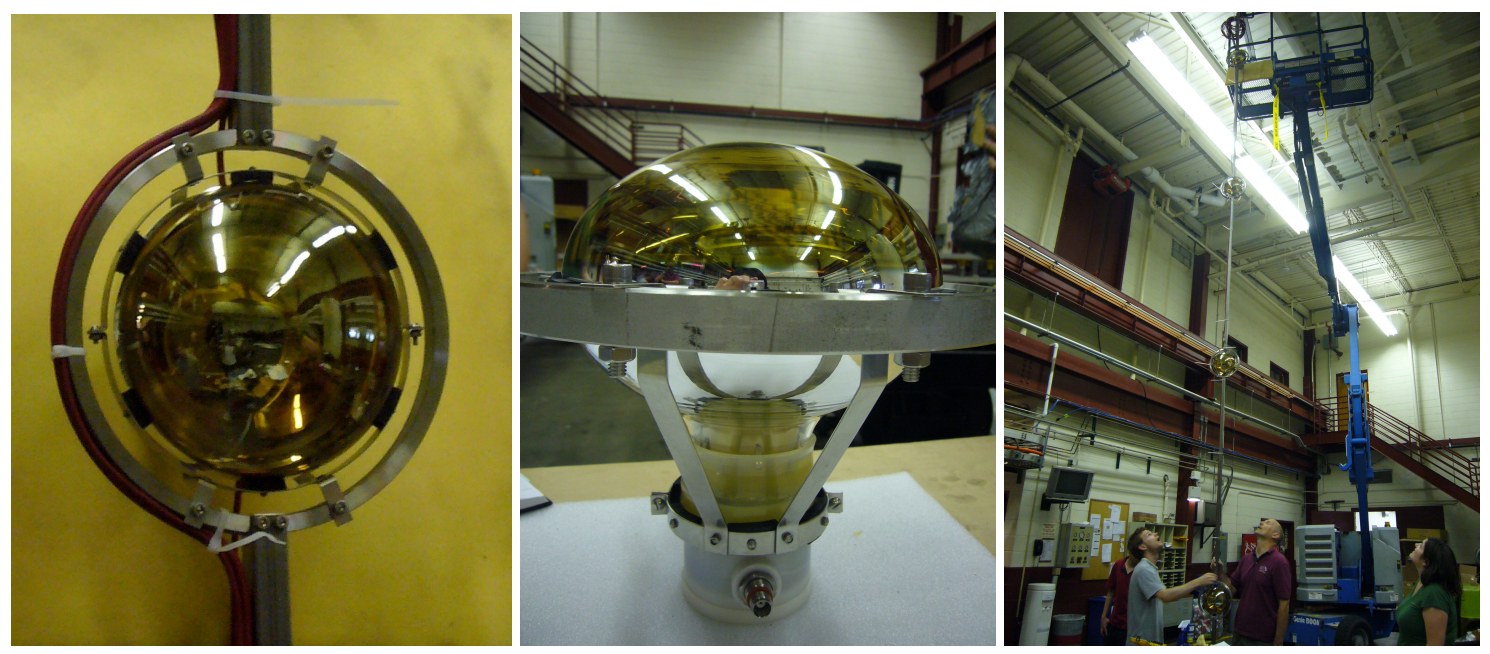

Figure 4. Veto PMT shown in mounting hardware (left, center), and assembled into a 4-PMT string (right).

\section{Electronics design of the MiniCLEAN veto subsystem}

The electronic components of the veto subsystem are the PMT high voltage supply, the PMT signal electronics, and the data acquisition electronics. A schematic showing the system outline is shown in Figure 5 .

The 48 veto PMTs use 6 channels of a 12-channel card of the MiniCLEAN inner liquid argon detector PMT high voltage supply (LeCroy 1461 VISyN) with SHV connectors. The voltage divider circuits to distribute power from each of $6 \mathrm{HV}$ channels to 8 PMTs are part of the amplifier discriminator card described in Section 3.1. The veto PMT signal electronics consist of 6 amplifier discriminator boards which each service 8 PMTs and 7 summer boards designed and built by MIT Bates Research and Engineering Center. The veto PMTs use 6 channels on one CAEN V1720 digitizer card of the MiniCLEAN DAQ system. The veto signal multiplexing electronics are part of the summer card described Section 3.3. The CAEN DAQ is described further in [5]. 


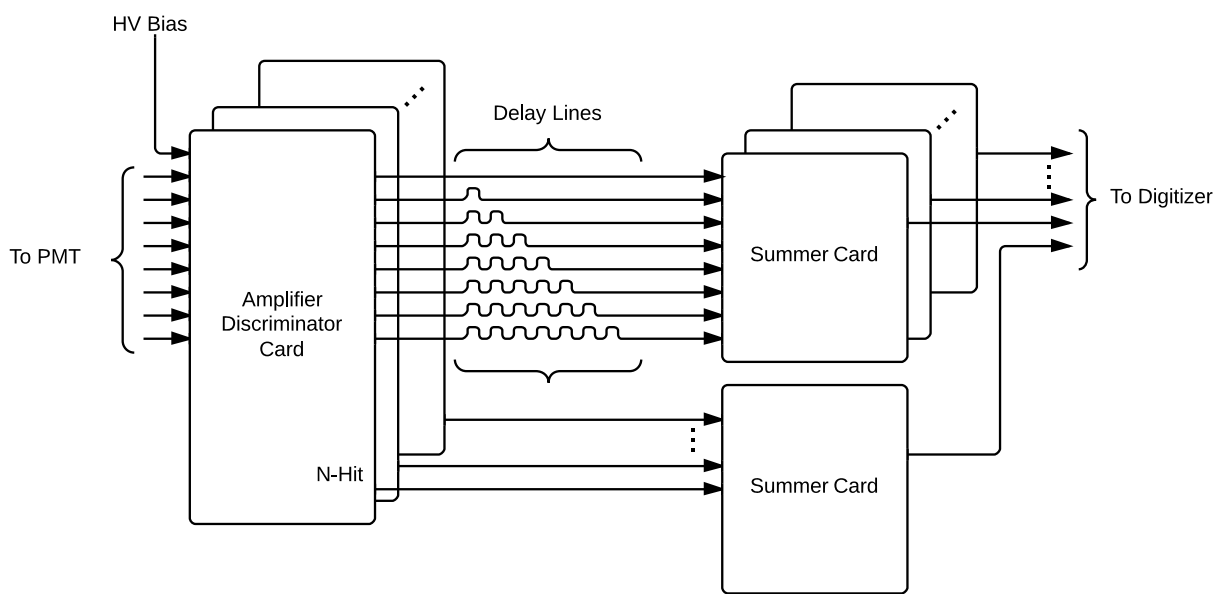

Figure 5. Block Diagram of MiniCLEAN Veto Electronics

\subsection{Amplifier discriminator board}

The amplifier discriminator board is the front end electronics board. The board provides high voltage distribution to the PMTs, signal amplification, and signal discrimination for the N-Hit sum. The PMT bias voltage is fed to each of the PMT connections through a single SHV connector. Each PMT capacitively couples the signal onto the bias voltage line. On the amplifier discriminator board each channel has an amplifier input capacitively coupled to a PMT bias line. The schematic for the PMT bias scheme, along with two channels of the amplifier discriminator, is shown in Figure 6 .

The HV bias components were surface mount types. Because of the aspect ratio of the width and length of some of the components, the components had to be raised slightly off of the board during the soldering process to ensure that flux could be cleaned from underneath the components. In addition, the HV components were potted with corona dope after assembly.

A high bandwidth current feedback amplifier (CFA) provides the large gain and bandwidth needed to amplify the pulses by a factor of 10 in a single stage. We selected the Analog Devices AD8000 CFA to amplify the PMT pulses. The amplified pulses are fed out to the delay lines and to the on-board comparators. Due to the high gain requirements, and the variations between the CFAs, all of the parts installed were tested beforehand and binned into parts with a similar DC offset. During board assembly, care was taken to ensure that all of the amplifiers installed on a single board had a DC offset which varied by no more than $10 \%$.

The comparator compares each channel with a programmable threshold voltage, common to all 8 channels on each board. The output of the comparator is a fast differential PECL output. These are summed with a differential amplifier and output using a transformer as a single ended signal, the "N-Hit sum" for those 8 channels.

The amplifier discriminator board has local linear power regulation. Each board has two $+5 \mathrm{~V}$ supplies, one $-5 \mathrm{~V}$ supply, and one $+3 \mathrm{~V}$ supply. The CFA runs on the $+5 \mathrm{~V}$ and $-5 \mathrm{~V}$ supplies. The comparator runs on the second $+5 \mathrm{~V}$ supply and the $+3 \mathrm{~V}$ supply (necessary for PECL output). The two $+5 \mathrm{~V}$ supplies isolate the output supply for the comparator in order to reduce cross talk. The regulators are fed by a common bipolar supply and ground nominally at $\pm 9 \mathrm{~V}$. The nominal values 


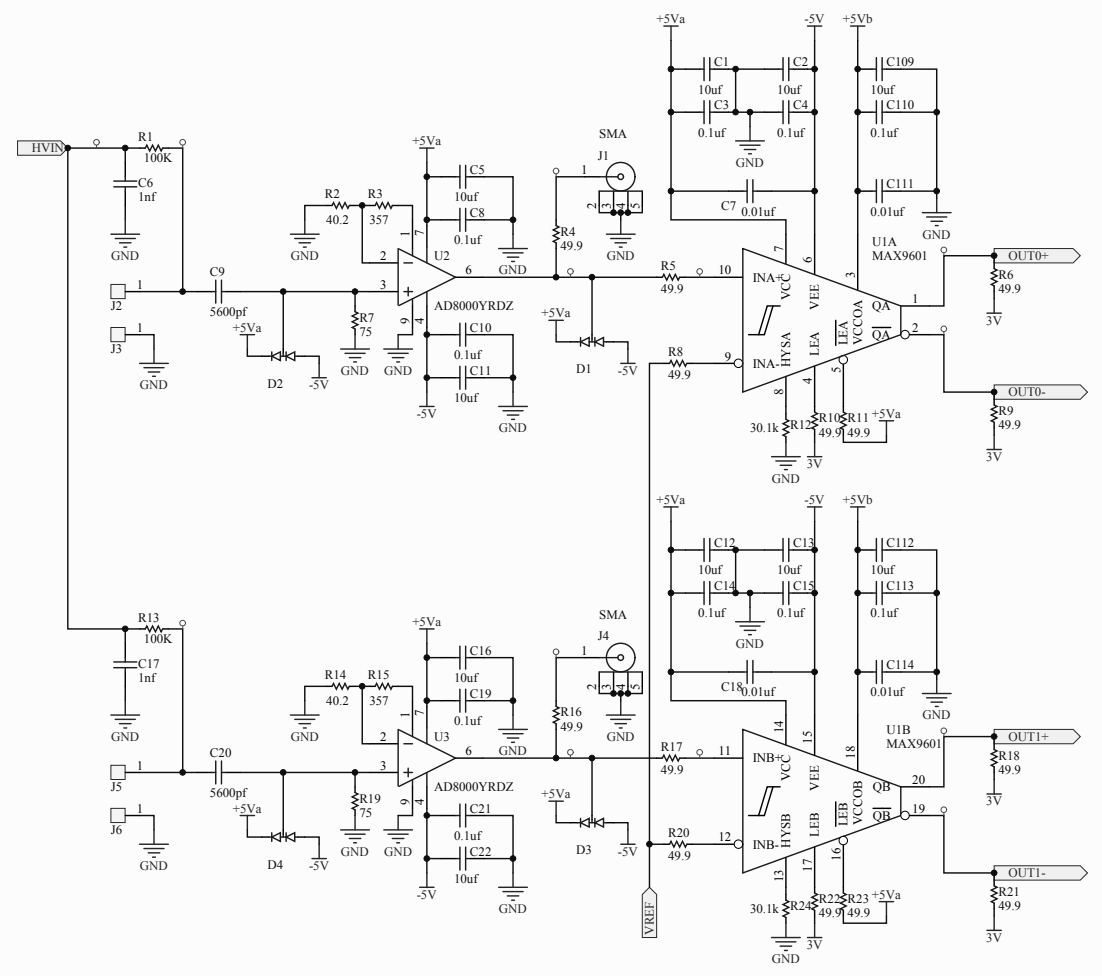

Figure 6. Two channels of the amplifier discriminator card. J2 and J5 make the signal connections to the PMTs. J1 and J4 make the connections to the delay lines. U2 and U3 are the amplifiers, U1 is the two channel comparator.

for current draw are $500 \mathrm{~mA}$ on the positive rail and $250 \mathrm{~mA}$ on the negative rail.

The board is constructed with 4 copper layers and standard FR4 substrate material, with 0.062 inch thickness. It is mounted on a piece of FR4 to protect HV nets on the bottom of the board from arcing and to fit it into a 6U VME style crate. 9 SHV connectors are mounted on a front panel connected to the FR4 and provide the HV bias input and eight PMT connections. These PMT connections attach on the left side of the board with short insulated wire pig tails. On the right side of the board 9 SMA connectors are board mounted. The SMA connectors provide the 8 signal outputs to the delay lines and the N-Hit sum signal. A photo of the amplifier discriminator board is shown in Figure 9 .

\subsection{Delay lines}

The delay lines connect from the amplifier discriminator boards to the summer boards. They are designed to delay the signals between $50 \mathrm{~ns}$ and $350 \mathrm{~ns}$. Since the signal pulses are expected to be short in comparison to the $50 \mathrm{~ns}$ delay window, delaying the signals and summing them together has the effect of time multiplexing the signals. The delay lines are made of different lengths of Belden 9310 cable fitted with male SMA connectors. The cable was chosen for its low attenuation, slow signal propagation speed, and small bend radius. There are 7 different lengths ranging form $32 \mathrm{ft}$ to $224 \mathrm{ft}$, with every $32 \mathrm{ft}$ equating to about $50 \mathrm{~ns}$ of delay. These are coiled inside a metal 
box with pig tails to connect to the amplifier discriminator boards and the summer boards. The metal box satisfies fire protection concerns in the mine, as the Belden 9310 is not plenum rated. There are two sets of each of the seven lengths in each box. One of the signals has a $\sim 0$ ns delay and is connected from the amplifier discriminator card to the summer card with a very short jumper cable. Figure \ shows the time multiplexed ouput of 8 channels fed with a synchronous pulse.



Figure 7. Oscillicope capture from the output of a summer board. Eight channels pulsed with a $10 \mathrm{mV}$ synchronous pulse into the amplifier discriminator board. The amplified signals are routed through the delay lines into the summer board. The top trace is the output of the summer board. The bottom trace is the N-Hit sum output from the amplifier discriminator board, used as a trigger.

\subsection{Summer board}

The summer board sums the analog channels after they have been delayed and also sums the $\mathrm{N}$ Hit sums from each of the 6 cards to make a global N-Hit sum. The summer board has 8 inputs which are female SMA connectors. Each of these inputs is fed, DC coupled, into a CFA to provide buffering. This also provides the opportunity to fine tune the gain and equalize the signals after the cable delay by adjusting the two feedback resistors. the outputs are fed into another CFA configured as an analog summing node. This outputs through a series resistor to an female MCX connector. A short jumper cable connects the summer board output to the digitizer input. Like the amplifier discriminator board, the summer board has local power regulation. Each board has one $+5 \mathrm{~V}$ supply and one $-5 \mathrm{~V}$ supply. The regulators are fed by a common bipolar supply nominally at $\pm 9 \mathrm{~V}$. The nominal values for current draw are $250 \mathrm{~mA}$ on the positive rail and $250 \mathrm{~mA}$ on the negative rail. A photo of the summer board is shown in Figure 9 , an example test pulse run through the summer board is shown in Figure 8 .

\subsection{Housing and assembly}

The electronics are housed in a rack which holds a crate for the electronics, a fan unit for cooling, a power supply unit, and all of the delay lines. The power supply module provides $\pm 9 \mathrm{~V}$ up to $130 \mathrm{~W}$. Estimated total power draw is $72 \mathrm{~W}$. The power supply uses two discrete $9 \mathrm{~V}$ switching power supplies which provide $+9 \mathrm{~V}, 0 \mathrm{~V}$, and $-9 \mathrm{~V}$ power rails running on the back of the rack. Connectors are attached to these rails and insert into each of the electronics boards. Each of the 

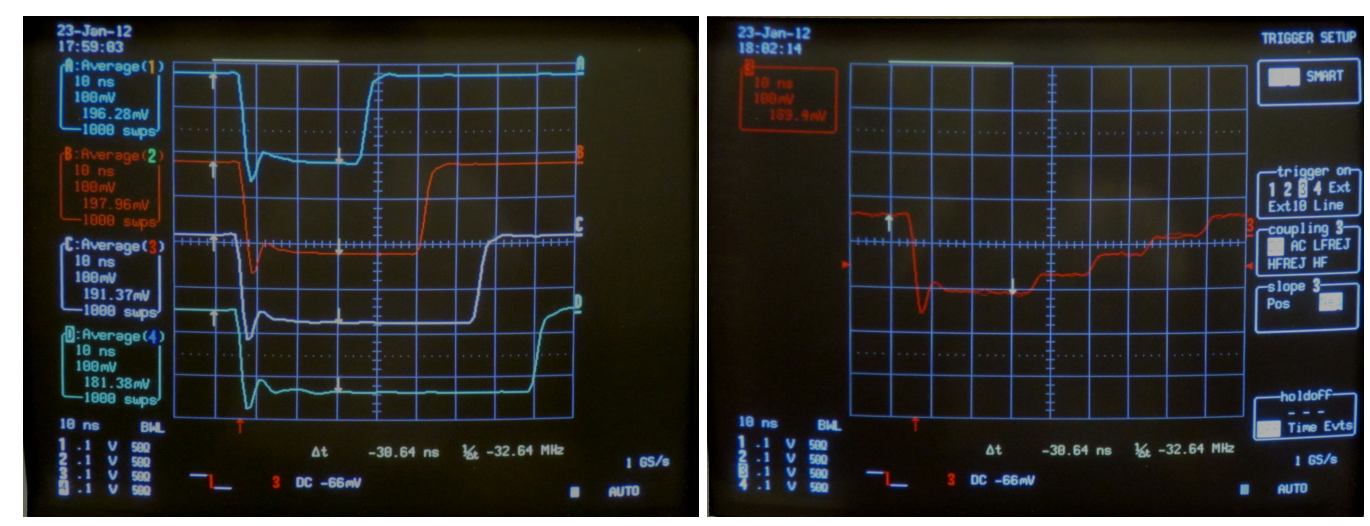

Figure 8. Input (left) and output (right) to the summer board measured with a test pulse generator.



Figure 9. Amplifier discriminator board (left) and summer board (right).

components was tested individually at MIT. Amplifier discriminator boards were high-pot tested and all channels were tested to ensure uniformity. Summer boards were similarly tested. The boards were assembled into a VME style crate and mounted in a rack together with the power supply unit, a fan unit, and the delay lines. An integration test was done with the MiniCLEAN electronics at Boston University to demonstrate the system running while attached to the CAEN DAQ system.

\section{Summary}

A full test of the electronics chain using simulated signals, integrated with the MiniCLEAN DAQ, demonstrated the veto system working properly. We were able to see simulated events pushed into 
the system and see the N-Hit trigger signal increase proportionally to the number of PMTs which were hit. We could observe the output signals on the time multiplexed outputs from the summer boards (Figure \). Once operational, the full system will multiplex 48 PMT signals into 6 digitizer channels and provide a system wide N-Hit sum. The system wide N-Hit will provide the veto trigger to the MiniCLEAN DAQ. The system is now at SNOLAB ready for implementation in the experiment.

\section{Acknowledgments}

The authors would like to acknowlege support from NSF grant PHY-0970047 and the MIT Bates Research and Engineering Center.

\section{References}

[1] M. Ronquest, The MiniCLEAN single-phase noble liquid dark matter experiment, IEEE Nucl.Sci.Symp.Conf.Rec. (2010) 1866-1872

[2] B. Aharmim et al., Measurement of the cosmic ray and neutrino-induced muon flux at the Sudbury Neutrino Observatory, Phys. Rev. D80 (2009) 012001

[3] D. Mei and A. Hime, Muon-induced background study for underground laboratories, Phys. Rev. D73 (2006) 053004

[4] J. Allison et al. Geant4 developments and applications, IEEE Transactions on Nuclear Science 53 No. 1 (2006) 270-278

[5] D. E. Gastler, Design of single phase liquid argon detectors for dark matter searches, Boston University Ph.D. Thesis (2012) 